\title{
Scattering in the inner accretion disk and the waveforms and polarization of millisecond flux oscillations in LMXBs
}

\author{
S. Y. Sazonov ${ }^{1,2}$ and R. A. Sunyaev ${ }^{2,1}$ \\ 1 Space Research Institute (IKI), Profsouznaya 84/32, 117810 Moscow, Russia \\ 2 MPI für Astrophysik, Karl-Schwarzschild-Str. 1, 86740 Garching bei München, Germany
}

Received 20 November 2000 / Accepted 23 April 2001

\begin{abstract}
The scattering by the inner accretion disk of X-ray radiation generated near the surface of a spinning neutron star in a low-mass X-ray binary (LMXB) has observable effects on the waveforms of millisecond X-ray flux oscillations produced e.g. during type-I bursts or in the millisecond pulsar SAX J1808.4-3658. We study these effects in the framework of a simplified model in which there is a single emitting spot on the stellar surface, which is visible both directly and in X-rays scattered from the disk. The main signature of scattering from a thin disk is that the pulse of scattered flux leads (if the star rotates in the same sense as the disk) or lags (in the contrary case) the primary pulse of direct emission by a quarter of a spin cycle. This is caused by Doppler boosting of radiation in the sub-relativistic Keplerian flow. The disk-scattered flux is revealed better in energy-resolved waveforms and the phase dependence of the polarized flux component. The phenomenon discussed permits direct testing of the presence of standard thin disks near the neutron stars in LMXBs and should be observable with future X-ray timing experiments having a few times better sensitivity than RXTE and also with sensitive X-ray polarimeters.
\end{abstract}

Key words. accretion, accretion disks - polarization - radiative transfer - binaries: close - stars: neutron X-rays: bursts

\section{Introduction}

The inner regions of the accretion disk around a weakly magnetic neutron star in a low-mass X-ray binary (LMXB) system intercept and re-emit a significant fraction of radiation generated near the surface of the neutron star. About half of the X-ray luminosity liberated via accretion originates in a boundary layer of the disk or in a spreading layer on the stellar surface. During type-I $\mathrm{X}$-ray bursts, the source luminosity almost entirely originates on the stellar surface. Therefore, scattering of the central emission in the inner disk should always have an important effect on the properties of the observed X-ray signal (Lapidus \& Sunyaev 1985).

The Rossi X-ray Timing Explorer (RXTE) detections of millisecond X-ray flux oscillations from many sources have demonstrated that the neutron stars in LMXBs are rapidly spinning, with frequencies ranging between 300 and $600 \mathrm{~Hz}$ (see Strohmayer 2000; Van der Klis 2000 for reviews). These oscillations are likely the result of azimuthal asymmetry in the generation of X-rays, e.g., there is a single emitting spot on the star (see Fig. 1). In future $\mathrm{X}$-ray timing experiments, a wealth of information on the geometrical and physical properties of neutron stars

Send offprint requests to: S. Sazonov, e-mail: ss@hea.iki.rssi.ru and the inner accretion flows in LMXBs will be obtained from high-quality measurements of oscillation waveforms. These can be non-sinusoidal for several reasons. First, if one considers the situation (as is frequently done) in which there is no accretion disk near the neutron star, then only half (neglecting aberration of light) of the surface of the spinning star can be visible at any instant. Therefore, periodic eclipses of the emitting zone may take place. Second, since the spin velocity at the stellar equator is of the order of $0.1 c$ (the speed of light), the emission pulse should be asymmetric due to Doppler boosting (the spot repeatedly moves towards and away from us - see Fig. 1; Chen \& Shaham 1989; Weinberg et al. 2001). Both general relativistic light bending and finite spot size act to reduce oscillation amplitudes (Pechenick et al. 1983; Wood et al. 1988; Riffert \& Meszaros et al. 1988; Weinberg et al. 2001).

If we take into account the accretion disk lying near the neutron star, it will still be possible for the above phase-dependent effects to take place, but additional effects will appear at the same time. In this paper we study the role of the accretion disk in forming oscillation waveforms. More specifically, we show that there are clear observational effects that allow us to test the presence of a standard thin disk near neutron stars in LMXBs. First, such a disk screens half of the star from our view. Second, if the emitting zone is located above the disk (with respect 


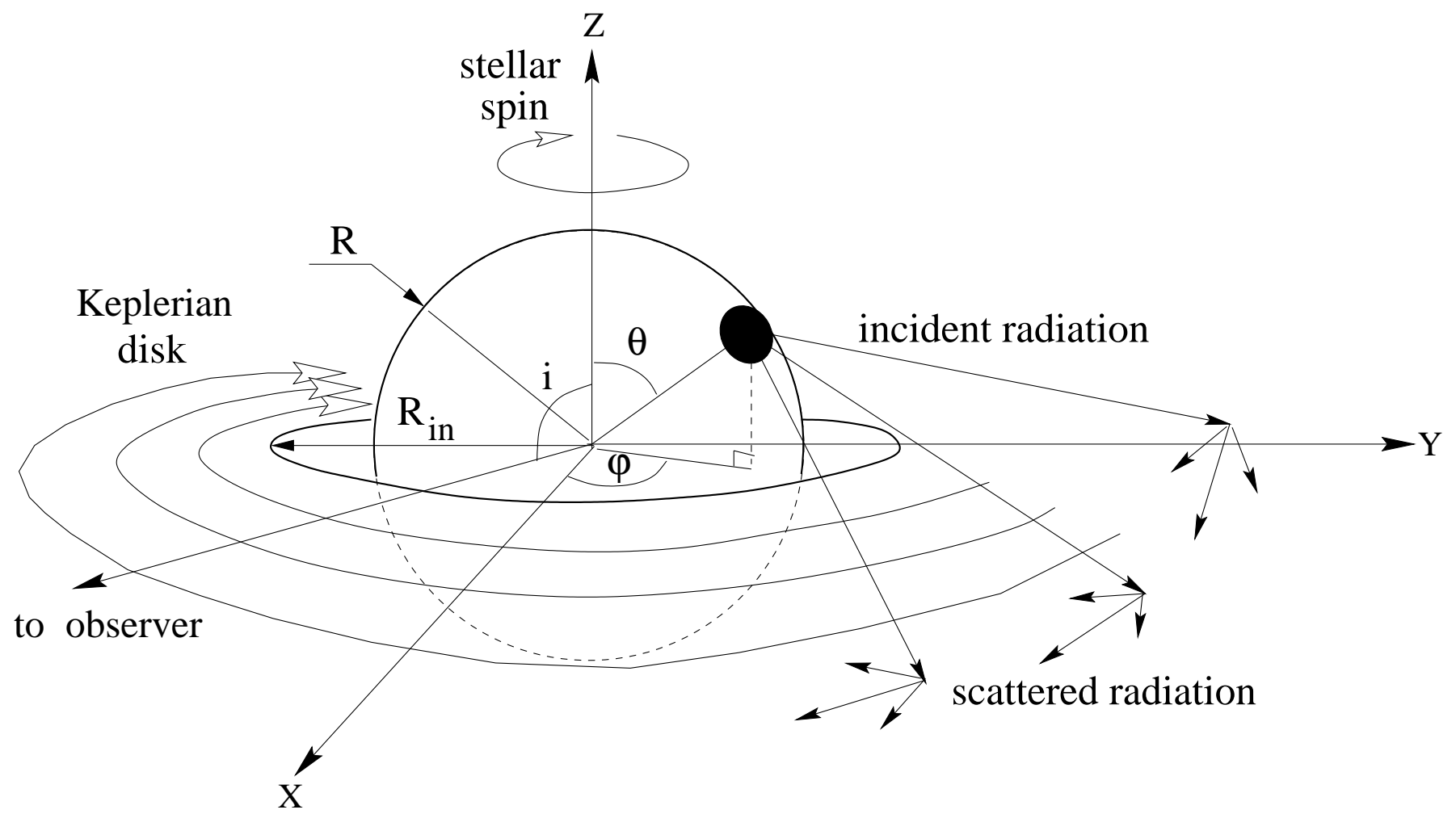

Fig. 1. Schematic picture of scattering of radiation emitted by a spot on the surface of a spinning neutron star from a surrounding Keplerian accretion disk.

to us), part of its flux is scattered by the disk in our line of sight.

In the innermost regions of the disk, matter is rotating with speeds $\sim 0.5 c$. Therefore, scattered photons are Doppler-boosted along the direction of the disk rotation: i.e., towards us when the bright spot is irradiating the sector of the disk in which matter approaches us (which approximately corresponds to the situation presented in Fig. 1), and away from us when the opposite sector of the disk is being irradiated (half a cycle later). As a result, the phase dependence of the scattered flux component will be distinctly different from that of the direct emission of the spot. Namely, the pulse of scattered emission will lead the pulse of direct flux by roughly a quarter of a cycle. Furthermore, X-ray flux registered during this secondary pulse will be substantially harder and more polarized (in absolute units) than at other phases (Fig. 2a). These conclusions only apply to binaries in which the neutron star rotates in the same sense as the disk. If the star rotates in the opposite sense (Fig. 2b), the scattered component will lag the direct signal, by the same quarter of a cycle. This makes it possible to uncover counterrotating LMXBs (see Sibgatullin \& Sunyaev 2000 on possibilities of formation of such systems).

General relativity predicts the existence of a gap between the last stable Keplerian orbit and the stellar surface for some (soft) neutron star equations of state (see, e.g., Kluzniak \& Wilson 1991). In geometries with a gap, part of the lower stellar hemisphere is visible, and also less flux from the upper hemisphere is scattered in the disk.
However, recent calculations (Sibgatullin \& Sunyaev 2000) show that there will be no gap if a neutron star of $1.4 M_{\odot}$ mass rotates with frequency $\sim 600 \mathrm{~Hz}$ in the same sense as the disk. For the slower rotation at $300 \mathrm{~Hz}$, a thin gap can exist, but its width proves to be smaller than the height of the boundary/spreading layer for the accretion rates typical of LMXBs (Inogamov \& Sunyaev 1999; Popham \& Sunyaev 2001). On the other hand, a large gap can arise in the case of counterrotation. We discuss some consequences of the presence of a gap in Sect. 3.2.

A similar situation probably takes place in the millisecond pulsar SAX J1808.4-3658, a unique object that demonstrates coherent $2.5 \mathrm{~ms}$ flux oscillations (Wijnands \& Van der Klis 1998; Chakrabarty \& Morgan 1998; Gilfanov et al. 1998). In this system, matter supposedly flows towards two nearly antipodal magnetic polar caps on the neutron star, and the accretion disk terminates at some distance from the stellar surface. Then, the hot spot located below the disk could be observable. However, the measured nearly sinusoidal pulse profile (Wijnands \& Van der Klis 1998) suggests that this spot contributes very little, if any, to the total signal. This fact can be used for constraining the geometry and inclination of the system. The upper hot spot should be observable both directly and in X-rays scattered from the disk.

Our scenario may apply to type-I X-ray bursts. Indeed, RXTE detections of nearly coherent oscillations during bursts from eight sources (see Strohmayer 2000 for a review; Wijnands et al. 2001; Galloway et al. 2001) imply the presence of a localized emitting zone. Apparently, this 

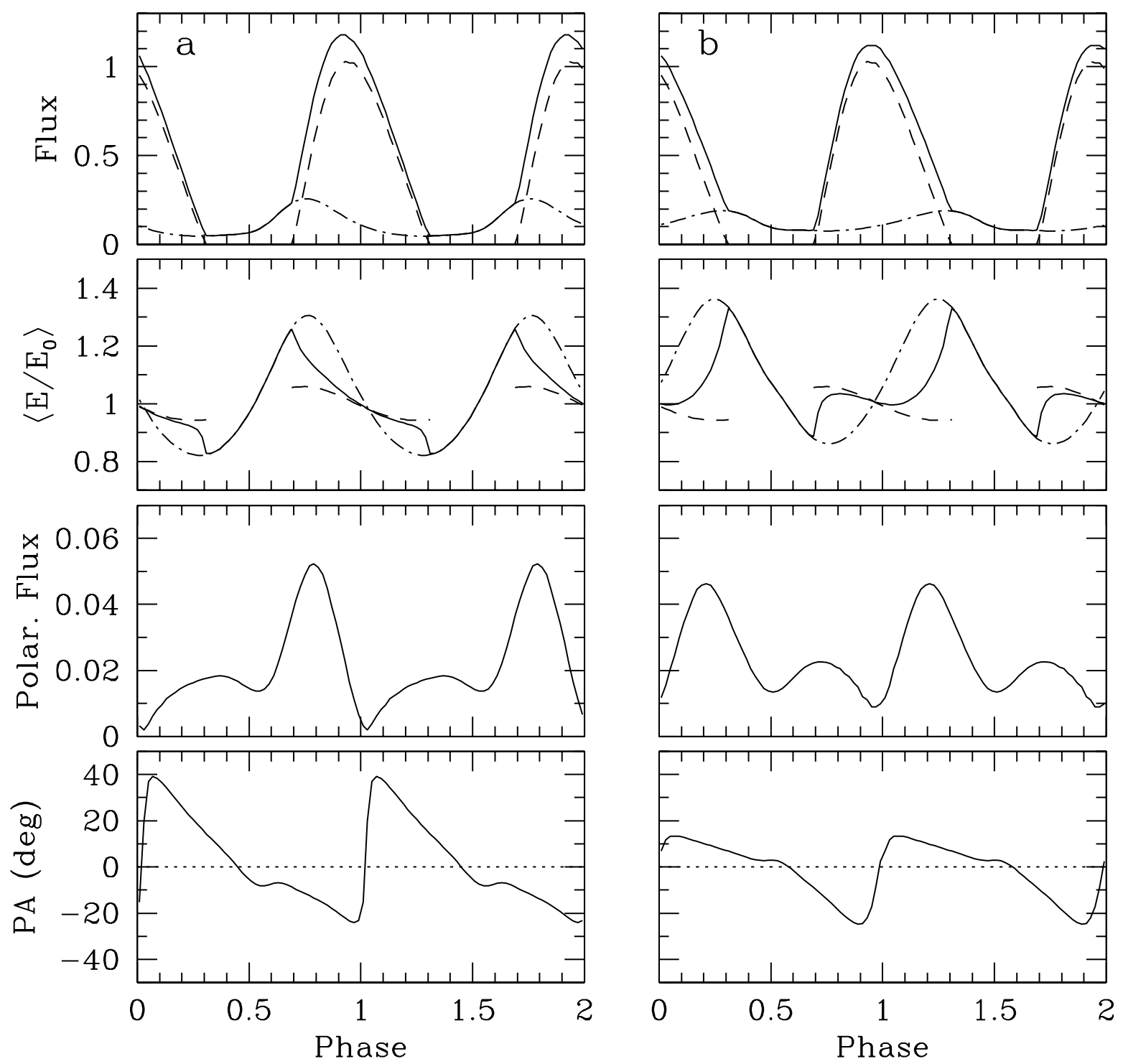

Fig. 2. a) Spin-phase dependences of various characteristics of emission from a LMXB, taking into account the effect of diskscattering, for the basic model $\left(R=R_{\text {in }}=6 G M / c^{2}\right), i=60^{\circ}, \theta=60^{\circ}$, a spin frequency of $f=300 \mathrm{~Hz}$, a point-like emitting spot $(\alpha=0)$ and corotation of the star and disk. The dashed curves correspond to direct emission from the spot, the dash-dotted lines to the disk-scattered radiation, and the solid lines to the sum of these two components. The panels from top to bottom are for: photon flux, mean photon energy increment, polarized flux (in the same units as total flux) and polarization angle. Note that only the scattered component contributes to the polarized flux. b) Same as a), but for the case of star-disk counterrotation.

zone slowly evolves in time (on sub-second timescales $\gg$ the spin period), as the thermonuclear burning front propagates over the stellar surface. It is not clear whether the innermost regions of a standard accretion disk can survive during a burst, when the disk is subject to strong radiation forces. In particular, radiation drag causes the matter flowing from the disk to lose angular momentum and spiral inward (Miller \& Lamb 1993). Obviously, the disk has a greater chance of remaining intact during the rise and decay stages of a burst, when the emitted luminosity, $L$, is much less than the critical Eddington luminosity, $L_{\text {Edd }}$. Note that if the inner disk is destroyed during the maximum of the burst, it can reconstruct very quickly at some later moment when the burst emission has faded substantially, because the characteristic times for all processes taking place within several stellar radii from the star are much shorter than the typical burst duration ( $10 \mathrm{~s})$.

Spots of enhanced brightness may also arise in a spreading layer on the surface of a neutron star (Inogamov \& Sunyaev 1999). This layer rotates differentially, with the azimuthal velocity ranging between the Keplerian velocity $(\sim 0.5 c)$ at the equator of the star, and the stellar spin velocity $(\sim 0.1 c)$ near the edges of the layer. Under such circumstances, long-living whirls, analogous to typhoons, Jupiter's famous red spot, or "cat eyes", may regularly appear in two bright latitudinal belts within the layer that are located in the upper and lower hemispheres equidistantly from the equator. The velocity shear is largest in these belts. Their location is determined by the accretion rate, namely, they become more distant from the 
equator (thus closer to the poles) with increasing accretion rate. Also, instabilitites developing in the accretion disk can modulate the accretion rate through the neck of the disk into the spreading layer. In particular, magnetic-field loops originating in the disk due to Balbus-Hawley instability may penetrate into the layer. All these phenomena can lead to the formation of bright emitting zones, rotating faster than the star itself, with quasi-frequencies up to the Keplerian one of $\sim 2 \mathrm{kHz}$ (Sunyaev \& Revnivtsev 2000). If a spot emits a significant fraction of the source luminosity during several spin cycles, quasi-periodic flux oscillations will appear. Averaged (with a fixed period) waveforms of such incoherent oscillations will tend to be nearly sinusoidal. However, the disk-scattered flux should still manifest itself clearly in the power density spectrum of the total signal (i.e., in the ratio of the powers contained in different quasi-harmonics), as well as in the phase delays $(\$ 0.25)$ between waveforms measured in different energy bands and in the phase shift $(\sim 0.25)$ of the polarized flux component with respect to total flux.

It is important to note that the considerations above are only applicable to atoll sources, i.e., to LMXBs with luminosities between 0.01 and $0.5 L_{\mathrm{Edd}}$. In this case, the approximation of a thin accretion disk (Shakura \& Sunyaev 1973) is appropriate. At luminosities approaching $L_{\mathrm{Edd}}$, like those of $Z$-sources, the boundary layer extends radially more than one stellar radius and is geometrically thick (Popham \& Sunyaev 2001). The adjacent disk also becomes thick. Therefore, our picture is no longer valid. Similarly in the case of $L<0.01 L_{\mathrm{Edd}}$, Compton cooling becomes inefficient for matter in the disk, and a geometrically thick advection-dominated accretion flow may form (e.g., Narayan et al. 1998).

\section{Model}

\subsection{Parameters and physical assumptions}

Let a neutron star of mass $M=1.4 M_{\odot}$ and radius $R$ spin with frequency $f$ and be surrounded by an infinitely thin, flat accretion disk with inner radius $R_{\text {in }}$. As our basic model, we adopt the geometry in which $R=R_{\text {in }}=R_{0}=$ $6 G M / c^{2}=12.4 \mathrm{~km}$, i.e., the neutron star is the same size as the innermost Keplerian orbit that corresponds to the Schwarzschild metric.

The spin axis is normal to the accretion plane and makes an angle $i$ with the line of sight. Two types of stellar spin are considered: one in the same sense as the Keplerian flow (corotation) and the other in the opposite sense (counterrotation).

A single homogeneously bright spot, of radius $\alpha$, on the star emits unpolarized radiation in accordance with the Lambert law in its rest frame, i.e. $F(\mu) \sim \mu$ (where $\mu$ is the cosine of the angle between the normal to the spot and the viewing direction). The spot is centered at an angular distance $\theta$ from the pole located in the upper hemisphere (see Fig. 1).
We also performed several computational runs, without treatment of polarization effects, for a more realistic situation in which the spot emits as an electron-scattering atmoshpere, i.e., $F(\mu) \sim 3 \mu(1+2 \mu) / 7$. In this case, radiation emerges linearly polarized from the spot and is more beamed. We found that the effect of the additional beaming on the phase dependence of flux (including the disk-scattered component) is fairly small and likely of the same order as the neglected effect of gravitational light bending (see below). The intrinsic polarization of the radiation emitted by the spot is also small for typical values of the angles $i$ and $\theta$, as follows directly from the classical results (Sobolev 1949; Chandrasekhar 1950). Indeed, the $\mu$-dependence of the degree of polarization, $P(\mu)$, has a sharp maximum of $11.7 \%$ at $\mu=0$. This maximum corresponds to a spot position at the limb of the star, when no flux is detectable. The polarized flux from the spot, i.e., $P(\mu) F(\mu)$, peaks in the course of stellar rotation when $\mu \sim 0.5$, and the maximum polarized flux, $F_{\max }^{\text {pol }}$, is typically only $\sim 1 \%$ of the maximum total flux, $F_{\max }$. The phase-dependent polarization effect due to disk-scattering proves to be much more significant (see Fig. 2), namely $F_{\max }^{\mathrm{pol}} / F_{\max } \sim 5 \%$ (for the sum of direct and scattered radiation) for typical values of $i$ and $\theta$. Based on these arguments, we restrict ourselves to considering below only the case of a spot emitting as a Lambert source.

Matter in the disk is in perfect circular Keplerian rotation; the velocity field (dependence on the radial coordinate $r$, as measured locally in the static frame) is calculated as appropriate for the Schwarzschild metric:

$v_{\phi}(r)=\left[2\left(\frac{3 r}{R_{0}}-1\right)\right]^{-1 / 2} c$,

so that $v_{\phi}\left(R_{0}\right)=0.5 c$.

The disk is perfectly reflective, which is a reasonable approximation for its inner region. In fact, even if $\mathrm{H}$-like and He-like ions of iron are abundant in the inner disk, these can only effectively absorb photons with energies lying in a narrow band above $9 \mathrm{keV}$. For softer X-ray radiation, the reflection albedo of the inner disk should be almost unity. Note also that the disk's surface layer having a Thomson depth $\tau \gtrsim 1$ is probably completely ionized due to irradiation by X-rays from the neutron star (e.g., Basko et al. 1974; Nayakshin et al. 2000). For the above reasons, the only radiation process we consider is Thomson scattering. Photons incident on the disk typically get scattered away after a few $(N<10)$ scatterings. With the temperature of matter in the disk $k T \sim 1 \mathrm{keV}$ (Shakura \& Sunyaev 1973) and for incident radiation with $E \lesssim 10 \mathrm{keV}$, the photon energy changes on average by a small fractional amount during the multiple scatterings: $|\Delta E / E| \sim\left|N(4 k T-E) / m c^{2}\right|<0.1$ [of course, the spectrum is also Doppler-broadened: $\left.(\Delta E / E)^{2} \sim 2 N k T / m c^{2}\right]$. For this reason, we consider scatterings to be coherent in the rest frame of the Keplerian flow. To calculate the angular distribution and polarization state of scattered radiation (Thomson scattering produces linear polarization) 
as measured in the disk rest frame, we apply the classical formulae of the theory of radiation transfer in planeparallel atmospheres for the Rayleigh angular scattering law (Chandrasekhar 1950).

General relativistic light bending is not taken into account. As a result, photon trajectories are straight lines, which greatly simplifies the computations. At the same time, this simplification cannot affect the main results of our study qualitatively. We do not consider the redshifting of radiation either, because its magnitude is the same (for the Schwarzschild geometry) for all photons, due to their common origin on the stellar surface.

All possible shadowing effects are taken into account. For the direct emission, these include: (1) the invisibility of the bright spot when it is turned away from the observer (provided that $i+\theta>90^{\circ}$ ), and (2) the screening by the disk $\left(\theta>90^{\circ}\right)$. The neutron star will, in turn, intercept part of the radiation scattered by the disk in the observer's direction if $i+\theta>90^{\circ}$ (the just quoted constraints are appropriate for the basic model with a point-like spot). Note that our model overestimates the duration of any eclipse intervals, due to the neglect of the light bending effect.

\subsection{The calculation procedure}

We introduce a reference frame, static with respect to the observer, with origin at the center of the neutron star (see Fig. 1). The accretion disk lies in the $X Y$-plane, and the $Z$-axis is the spin axis of the star. For a given inclination angle $i$, the observer is at infinity in the direction $\boldsymbol{l}=(\sin i, 0, \cos i)$. Instantaneous positions of the center of the emitting spot are defined by the azimuthal angle $\phi$, in addition to the previously defined parameter angle $\theta$.

The calculation essentially consists of following, both in space and time, the tracks of a large number of photons emitted by the bright spot. Multi-dimensional integration is carried out over $\phi$ (from 0 to $2 \pi$ ), as well as over many zones that compose the total area of the bright spot and the disk. The integration over the disk is limited radially by an outer boundary of $R_{\text {out }}=11 R_{0}$. Only a small fraction, $<10 \%$, of the total X-ray flux illuminating the whole disk is intercepted by yet farther regions (if the warping of the disk is neglected), causing their role in forming the scattered signal to be practically unimportant. It is also worth mentioning that for $\theta>60^{\circ}$ (which is expected to occur with $1 / 2$ probability if spot positions are random), more than $75 \%$ of the radiation incident on the disk is received by the innermost region of $R_{0}<r<4 R_{0}$.

On output, we obtain the arrival time, energy shift $\left(E / E_{0}\right)$, and polarization state for each tested photon. Energy shifts have a Doppler origin: either due to the stellar spin or due to the Keplerian rotation of the disk. Our code calculates the direct signal, the component scattered from the disk and the sum of these two. Several types of final results can be produced: (1) oscillation waveforms, i.e., the number of photons as a function of spin phase $\xi$ ( $\xi=$ arrival time/spin period), (2) the phase dependence of the average energy increment, $\left\langle E / E_{0}\right\rangle(\xi)$ (provided that the spot emits monochromatic radiation of energy $\left.E_{0}\right)$, (3) oscillation waveforms in several specified narrow energy bands for a given energy spectrum of the spot emission, and (4) the phase dependence of the polarized flux component (namely, amplitude and the position angle of the polarization plane).

The zero of $\xi$ is defined as follows. Suppose the bright spot is at some moment in the position $\phi=0$ and emits a photon in the direction $\boldsymbol{l}$ (along the line of sight). Imagine that at the same instant another photon is emitted from the center of the star, $\boldsymbol{r}=(0,0,0)$, also in the direction $\boldsymbol{l}$. We postulate that the second photon will arrive at the detector at phase 0 . This implies that the first photon will reach the detector a little earlier, at $\xi=1-R f c^{-1} \cos (\theta-i)$. Note that this phase difference, which has a time-of-flight origin, is small for $f<600 \mathrm{~Hz}$ : $1-\xi<0.025$. Larger phase delays of this type can arise for photons that experience a disk-scattering on their way to the observer. The Thomson-scattering process itself is assumed to introduce no extra phase delay, which is true for our case of a geometrically thin disk.

One phenomenon is worth mentioning here. As the neutron star spins, the zone of the disk that is illuminated by the bright spot rotates with a speed of $2 \pi f r$, which exceeds the speed of light far enough from the star. For example, at $f=600 \mathrm{~Hz}$, this occurs at $r>80 \mathrm{~km}$. Such distant regions, however, intercept very little of the spot emission. For this reason, the contribution of superluminal effects to the phase dependence of the scattered component is negligible.

Let us now turn to the main physical mechanism at work, namely, Doppler boosting of radiation, which operates both during emission from the spinning star and during scattering from the disk. The disk-related effect is the larger, as follows from a comparison of the Keplerian velocity, $\sim 0.5 c$, with the spin velocity at the stellar equator, $0.08(f / 300 \mathrm{~Hz}) c$ (as measured locally). Our code makes use of a set of equations giving the Lorentz transformations of photon energy $E$, direction of propagation $\boldsymbol{k}$, solid angle $\mathrm{d} \Omega$, and the electric $(\boldsymbol{E})$ and magnetic $(\boldsymbol{H})$ field vectors (Landau \& Lifshits 1975):

$$
\begin{aligned}
& E^{\prime}=E \gamma\left(1-\frac{\boldsymbol{k} \boldsymbol{v}}{c}\right) \\
& \boldsymbol{k}^{\prime}=\frac{1}{\gamma(1-\boldsymbol{k} \boldsymbol{v} / c)}\left\{\boldsymbol{k}+\left[\frac{c(\gamma-1)}{v^{2}} \boldsymbol{k} \boldsymbol{v}-\gamma\right] \frac{\boldsymbol{v}}{c}\right\} \\
& \mathrm{d} \Omega^{\prime}=\frac{1}{\gamma^{2}(1-\boldsymbol{k} \boldsymbol{v} / c)^{2}} \mathrm{~d} \Omega
\end{aligned}
$$

$$
\begin{gathered}
\boldsymbol{E}_{\|}^{\prime}=\boldsymbol{E}_{\|}, \quad \boldsymbol{E}_{\perp}^{\prime}=\gamma\left(\boldsymbol{E}+\frac{[\boldsymbol{v} \boldsymbol{H}]}{c}\right)_{\perp}, \\
\boldsymbol{H}_{\|}^{\prime}=\boldsymbol{H}_{\|}, \quad \boldsymbol{H}_{\perp}^{\prime}=\gamma\left(\boldsymbol{H}-\frac{[\boldsymbol{v} \boldsymbol{E}]}{c}\right)_{\perp},
\end{gathered}
$$


where $\gamma=\left[1-\frac{v^{2}}{c^{2}}\right]^{-1 / 2}$.

Here, the unprimed terms are measured in the observer's frame, whereas the primed ones in a moving (with velocity $\boldsymbol{v}$ ) frame, which can be either the rest frame of the bright spot or the rest frame of the Keplerian flow; the subscripts $\|$ and $\perp$ denote a vector's components along and normal to $\boldsymbol{v}$, respectively. Equations (5) govern the Lorentz transformation of the polarization plane. Note that the polarization degree is a Lorentz invariant (e.g., Landau \& Lifshitz 1975).

An individual disc-scattering act is computed in three steps. At first, the energy, $E_{0}$, the direction of propagation, $\boldsymbol{k}_{0}$, and the photon flux of the incident unpolarized radiation are transformed to the rest frame of the Keplerian flow, using Eqs. (2)-(4). Then, the photon flux emergent in the direction $\boldsymbol{l}^{\prime}$ and its polarization state are calculated in this frame, following Chandrasekhar (1950). Finally, the reverse transition to our frame is implemented, using Eqs. (2)-(5). The emergent photon energy can also be directly related to the incident energy:

$E=E_{0} \frac{1-\boldsymbol{k}_{0} \boldsymbol{v} / c}{1-\boldsymbol{k} \boldsymbol{v} / c}$

\section{Results}

\subsection{Point-like emitting spot}

We begin by considering the situation in which the bright spot is infinitely small. Most of our results are obtained for the basic model $\left(R=R_{\text {in }}=R_{0}\right)$ and $f=300 \mathrm{~Hz}$.

In Fig. 2, we plotted the phase dependences, which correspond to $i=60^{\circ}$ and $\theta=60^{\circ}$, of photon flux, mean energy increment (for an initially monochromatic spectral line), polarized photon flux and polarization position angle (which is measured relative to the meridional plane defined by the normal to the disk and the line of sight, and takes values between $-90^{\circ}$ and $90^{\circ}$ ). The cases of star-disk corotation and counterrotation are compared.

As expected, the pulse of direct emission is asymmetric, in particular, flux peaks at $\xi \approx 0.95$, rather than at $\xi=1$, which would be the case if the star were spinning very slowly. The maximum of $\left\langle E / E_{0}\right\rangle$ for the direct signal is at $\xi \approx 0.75$, which corresponds to the spot approaching us. These spin-induced Doppler effects are well-known (see, e.g., Weinberg et al. 2001). The spot is not visible directly during the eclipse centered at $\xi=0.5$. The direct emission is unpolarized by assumption.

As regards the scattered component, flux, mean energy increment and polarized flux all peak near $\xi=0.75$ (i.e., before the peak of direct emission) in the case of corotation, but near $\xi=0.25$ (i.e., after the main peak) in the case of counterrotation. The partial obscuration of the disk by the star, which occurs around $\xi=0.5$, plays a minor role for the given values of $i$ and $\theta$, but becomes more important for larger values of these angles. It is obvious from the phase dependences for the total signal (the

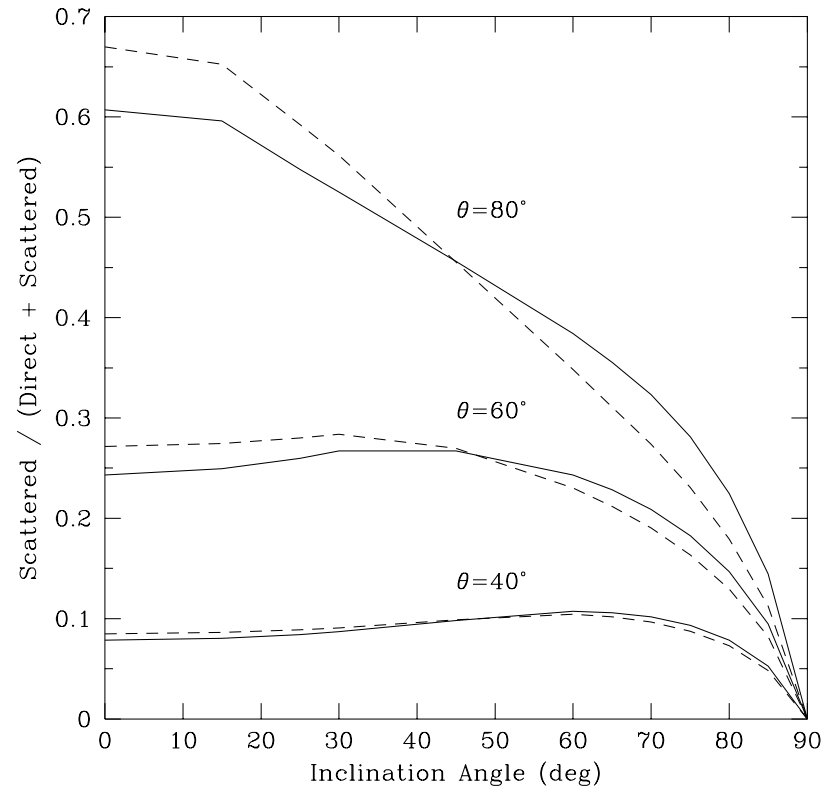

Fig. 3. Relative contribution of the disk-scattered component to the phase-integrated photon flux as a function of the angles $i$ and $\theta$, for the basic model with $f=300 \mathrm{~Hz}, \alpha=0$ and the case of corotation (solid lines). For comparison, the results for an imaginary non-rotating disk and very slowly spinning neutron star $(f=3 \mathrm{~Hz})$ are also shown (dashed lines).

sum of the direct and scattered components) shown in Fig. 2 that the presence of disk-scattered flux could be most clearly established by means of X-ray polarimetry.

The amplitude of the disk-scattering feature in an oscillation waveform is proportional to the contribution of scattered flux to the time-integrated signal, which can be found by integrating the phase dependence. In Fig. 3, the relative contribution of scattered flux is shown as a function of the inclination angle for three values of $\theta$, and $f=300$. These dependences are compared with the dependences that would result if the disk were non-rotating and the neutron star were spinning very slowly $(f \rightarrow 0)$, so that no Doppler boosting of radiation would take place. One can see that the differences between the two sets of curves are small, i.e., Keplerian motion has a much smaller effect on the integral of flux than on its phase dependence. The spin frequency proves to be yet less important here. We can draw the following conclusions from Fig. 3. The relative contribution of the scattered component is less than $10 \%$ for $\theta<40^{\circ}$. At lower positions (with respect to the disk) of the bright spot, this contribution can be much larger (up to $100 \%$ at $i=0, \theta=90^{\circ}$ ). Provided that bright spot positions are random, $60^{\circ}<\theta<90^{\circ}$ in $50 \%$ of situations. Similarly, $60^{\circ}<i<90^{\circ}$ for every second binary. Therefore, scattered emission should typically contribute $\sim 20 \%$ to the total signal.

Figure 4 is similar to Fig. 3, but for the polarization degree of the phase-integrated total flux. Typically, the polarization degree is $\sim 3 \%$ and is somewhat enhanced owing to the Doppler boosting of radiation in disk-scattering. Our results for a non-rotating disk and slowly spinning 


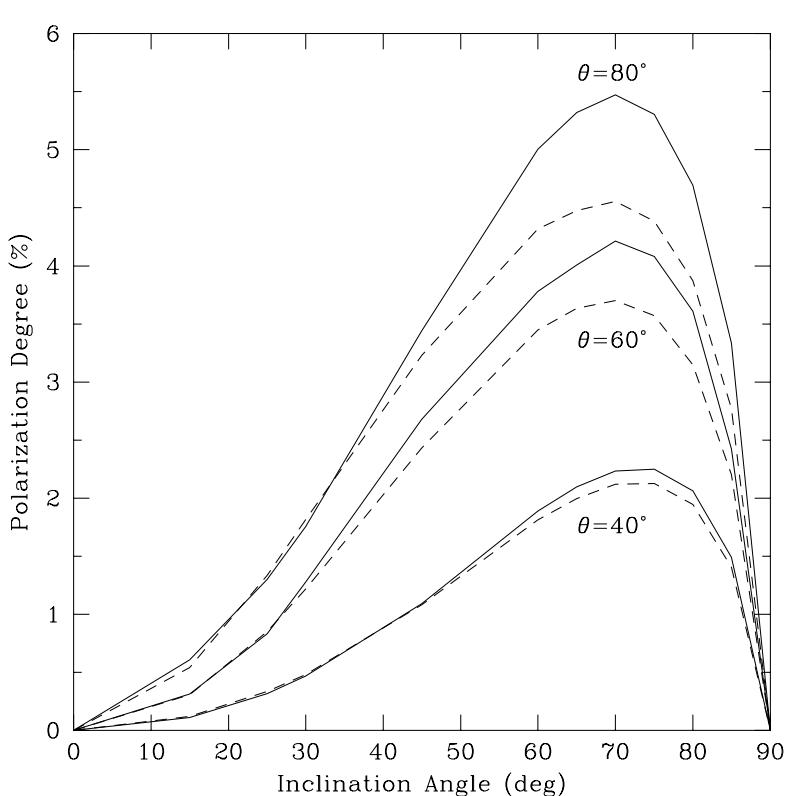

Fig. 4. Polarization degree of the phase-integrated total (direct plus scattered) flux as a function of the angles $i$ and $\theta$, for the basic model with $f=300 \mathrm{~Hz}, \alpha=0$ and the case of corotation (solid lines). For comparison, the results for an imaginary nonrotating disk and very slowly spinning neutron star $(f=3 \mathrm{~Hz})$ are also shown (dashed lines).

neutron star presented in Figs. 3 and 4 are in agreement with the results of Lapidus \& Sunyaev (1985).

Let us now examine the effect of the scattered component on the harmonic content of flux oscillations. To this end, oscillation waveforms need to be Fourier-transformed. In Fig. 5, we compare the rms-amplitudes of variability contained in the first two harmonics. Note that much of the harmonic content of the direct emission is due to the Doppler boosting by the spinning star; more details on this can be found in Weinberg et al. (2001). The scattered component generally causes the total signal to be more sinusoidal (i.e., the ratio of the amplitude of the second harmonic to that of the first harmonic decreases). This effect is mostly the result of the presence of scattered flux during eclipses of the bright spot (see Fig. 2) and becomes more pronounced for lower spot positions (see Fig. 5b).

\subsection{Effects of the disk/star size ratio, spin frequency and spot size}

How crucial is the condition $R=R_{\text {in }}=R_{0}$, which defines our basic model? In Fig. 6, three different oscillation waveforms of disk-scattered radiation are shown, all corresponding to $i=60^{\circ}$ and $\theta=80^{\circ}$. In one case, the geometry is standard. In the second case, the neutron star extends beyond the last stable Keplerian orbit: $R=R_{\text {in }}>R_{0}$. In the third case $-R=R_{0}<R_{\text {in }}$, which may approximately correspond to the millisecond pulsar SAX J1808.4-3658, a transparent zone exists between $R$ and $R_{\text {in }}$. One can see that the amplitude of the scattered component is smaller by a factor of 2 in the case of this gap geometry. The

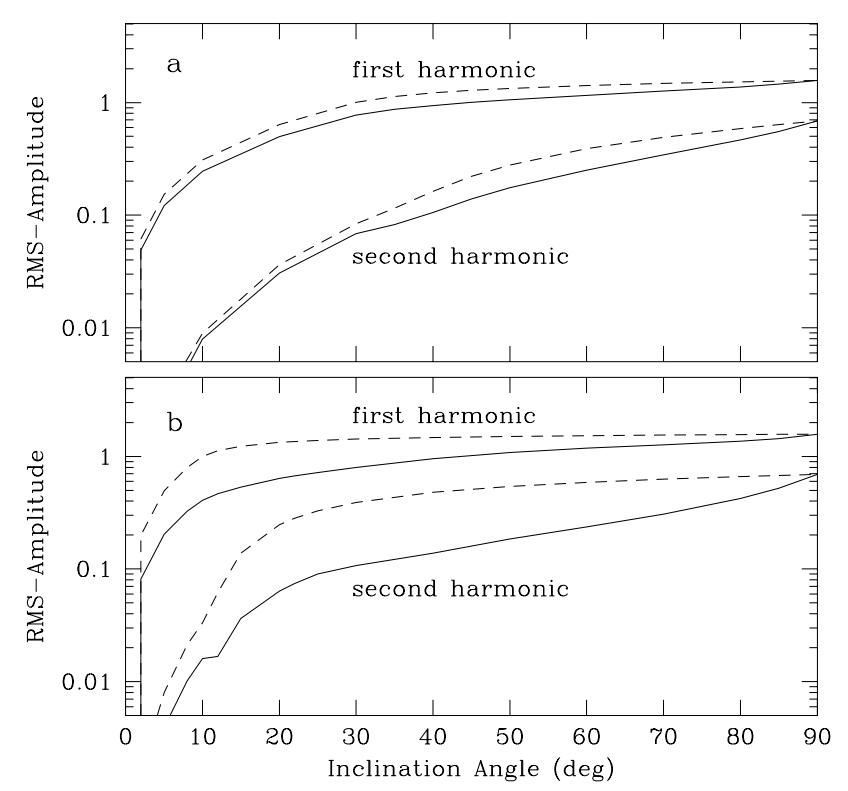

Fig. 5. Amplitudes of the first and second Fourier harmonics in the direct flux from the spot (dashed lines) and in the total signal (solid lines) as a function of the inclination angle $i$, for the basic model with $f=300 \mathrm{~Hz}, \alpha=0$ and the case of corotation. a) $\theta=60^{\circ}$, b) $\theta=80^{\circ}$.

waveforms for the "normal-size" and "oversize" neutron stars are only slightly different. We conclude that the oscillation phase dependence and amplitude are almost independent of the stellar size as long as $R=R_{\text {in }} \gtrsim R_{0}$. On the other hand, the scattered component is sensitive to the ratio $R_{\text {in }} / R$, particularly for near-equatorial spot positions, if a gap separates the star from the accretion disk.

Another parameter that can have an effect on oscillation waveforms is the spin frequency, $f$. In Fig. 7, we compare two cases: one of very slow rotation, $f=3 \mathrm{~Hz}$, and the other of fast rotation, $f=600 \mathrm{~Hz}$. In the first case, there is no Doppler boosting of the direct emission. In the second case, when the equatorial spin velocity is $0.16 c$, the resulting asymmetry is very pronounced, as the direct flux peaks at $\xi \approx 0.9$. However, the scattered component is relatively insensitive to $f$.

We have so far assumed that the bright spot is pointlike. In reality, radiation is generated over some extended region of the stellar surface. Let us examine the effect of the spot radius, $\alpha$, on oscillation waveforms. An example is presented in Fig. 8. A circular spot is centered at the equator, so that half of its area is above the disk, producing the direct and scattered flux components, while the lower half is invisible and has no effect on the phase dependence. As expected, waveforms become smoother with increasing $\alpha$. The asymmetry between the rising and declining branches of the pulse due to disk-scattering becomes less pronounced as $\alpha$ increases. As $\alpha$ approaches $90^{\circ}$, the waveform becomes nearly sinusoidal, and the role of the scattered component effectively reduces to shifting the pulse in phase. 


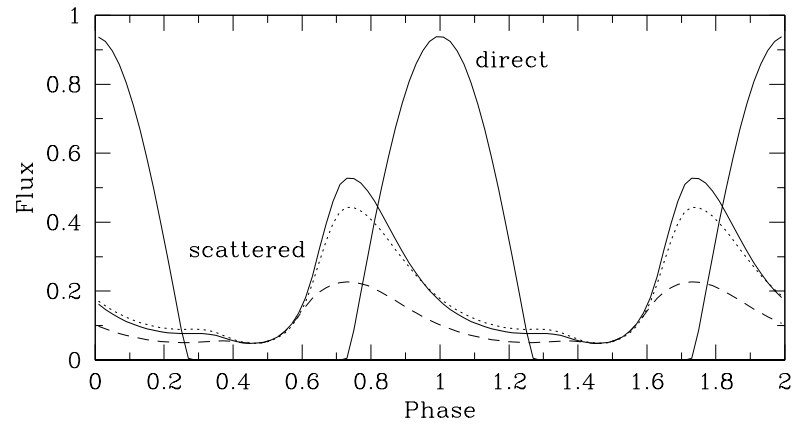

Fig. 6. Effect of the ratio of the disk inner radius, $R_{\text {in }}$, to the stellar radius, $R$, on the oscillation waveform of the diskscattered component, for $i=60^{\circ}, \theta=80^{\circ}, \alpha=0$ and the case of corotation. A low spin frequency, $f=3 \mathrm{~Hz}$, is taken, so that the Doppler boosting by the star is unimportant, and the direct signal (the solid line with the corresponding label) is independent of $R$. Three geometries are considered: (1) the basic model $\left(R=R_{\text {in }}=6 G M / c^{2}\right.$; solid line), $(2) R=R_{\text {in }}=$ $8 G M / c^{2}$ (dotted line) and (3) $R=6 G M / c^{2}, R_{\text {in }}=8 G M / c^{2}$ (dashed line).

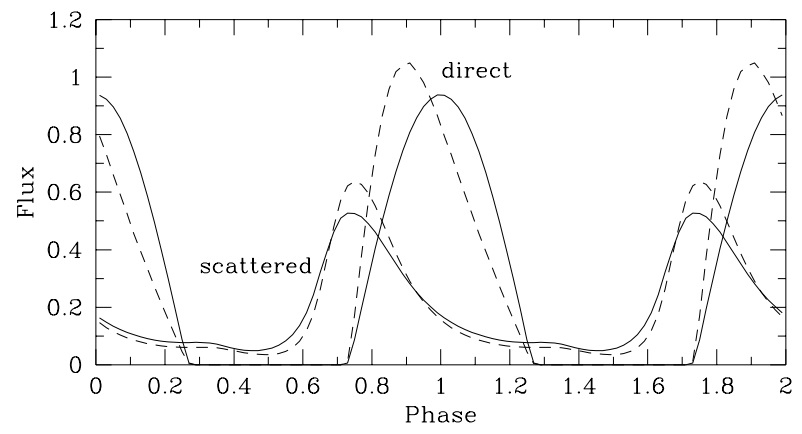

Fig. 7. Effect of the spin frequency, $f$, on the oscillation waveforms of the direct and scattered flux components, for the basic model with $i=60^{\circ}, \theta=80^{\circ}, \alpha=0$ and the case of corotation. Two cases are considered: one of very slow rotation $(f=3 \mathrm{~Hz}$; solid lines), and the other of fast rotation $(f=600 \mathrm{~Hz}$; dashed lines).

Figure 9 is similar to Fig. 8, but for the polarized flux component. From these two figures one can see that the pulse of polarized flux is always shifted relative to the pulse of total flux by approximately a quarter of a cycle, independent of how large the bright spot is. Whether this phase shift is negative or positive is determined by the sense of the stellar spin with respect to the disk rotation.

\subsection{Energy-resolved waveforms}

Photons arriving at the detector directly from the neutron star or upon scattering in the Keplerian disk have been Doppler-shifted in energy. The phase-dependent fractional shift, $\Delta E / E$, is $\sim 0.08(f / 300 \mathrm{~Hz}) \cos [2 \pi(\xi+0.25)]$ in the former case (for a spot at the stellar equator and an edgeon view of the binary), and $\sim 0.5 \cos [2 \pi(\xi \pm 0.25)]$ in the latter (where "+" in the argument of cos corresponds to the case of corotation). Therefore, energy-resolved oscillation waveforms can provide more detailed information

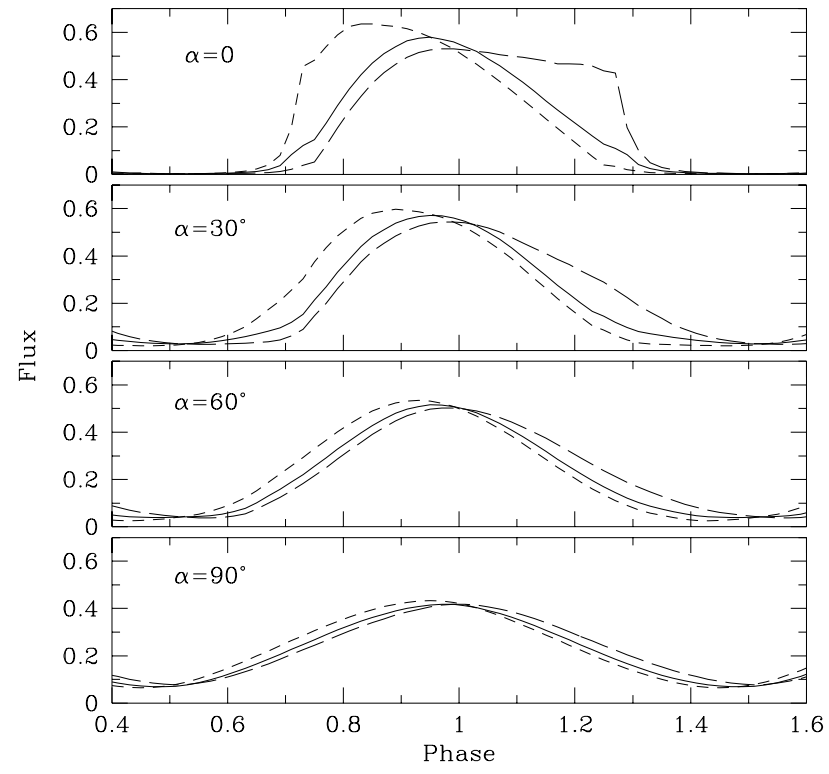

Fig. 8. Effect of the bright spot size on the oscillation waveform of total (direct plus scattered) flux, for the basic model with $i=60^{\circ}, \theta=90^{\circ}$ and $f=300 \mathrm{~Hz}$. Three cases are compared: of corotation (short-dashed lines), of counterrotation (long-dashed lines) and also of a non-rotating disk (solid lines). Note the different than in the other figures phase region, which permits a better view of the waveforms.

on the scattering of the central emission in the disk than just the phase dependence of total flux. As an example, we consider a situation in which a spot of radius $\alpha=60^{\circ}$, centered at the equator, emits black-body radiation with a temperature $k T=2 \mathrm{keV}$, a value typical for type-I bursts. We made computations for 5 energy bands covering the range $E<20 \mathrm{keV}$. The resulting oscillation waveforms are presented in Fig. 10. The role of the scattered component apparently becomes more important with increasing energy. Not only the relative contribution of scattered flux becomes larger, but also the pulse is much more asymmetric at high energies than at low.

To make the analysis more quantitative, we have Fourier-transformed the phase dependences shown in Fig. 10 and thus found the phase lag of the fundamental harmonic as a function of photon energy (Fig. 11). We see that the role of disk-scattering in "delaying" low-energy photons is more important than the corresponding role of stellar spin. This can be explained as follows. Both dependences plotted in Fig. 11 approach an asymptotic value of -0.25 as $E \rightarrow \infty$, because at $E \gg k T$ flux only arises at phases $\xi \sim 0.75$ (when both the spot and the illumination front on the disk are moving towards us). The phase lead will constitute a significant fraction of this 0.25 value for $E \gtrsim k T /\langle\Delta E / E\rangle$ [because of the $\exp (-E / k T)$ factor in the spectral law]. Therefore, in order to have a substantial hard-to-soft phase difference due to disk-scattering, one needs $E \gtrsim 2 k T$, as compared to $E \gtrsim 10(300 \mathrm{~Hz} / f) k T$ in the case of stellar rotation. Thus, in the latter case, large phase leads $(\sim 0.1)$ can only materialize very far in the Wien tail of a black-body spectrum, i.e., in a region 


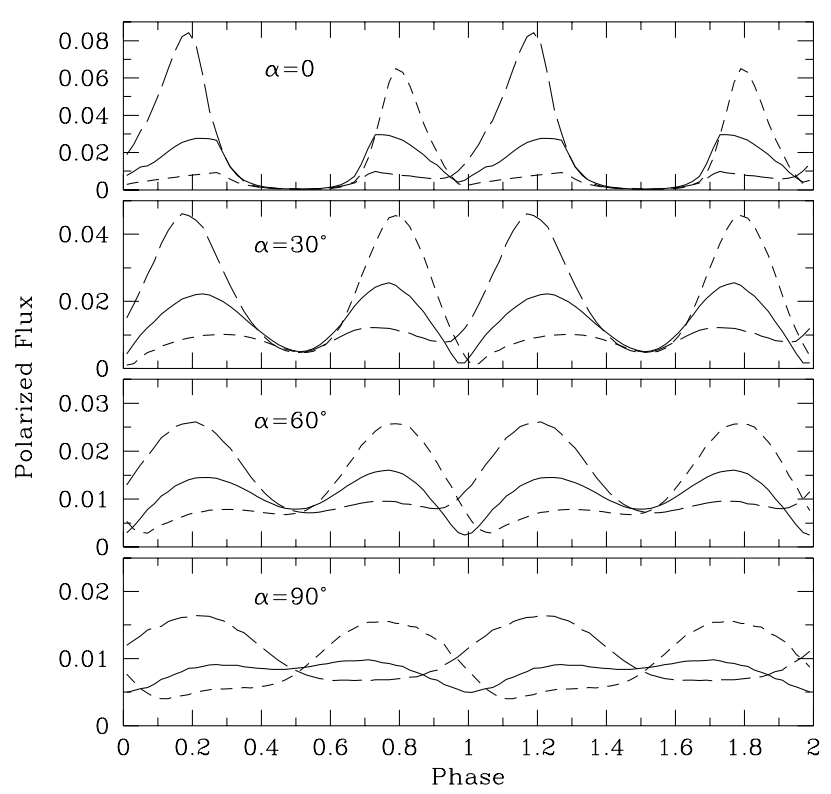

Fig. 9. Same as Fig. 8, but for the polarized flux component.

where virtually no emission is generated. Note that in the case of a counterrotating disk, hard emission will lag soft emission.

\section{Conclusion}

We have considered the effect of X-ray scattering by a thin accretion disk on the phase dependence of spin-modulated emission from a neutron star with a weak magnetic field. This effect makes it possible to test the presence of standard thin accretion disks around the neutron stars in LMXBs. The only other existing experimental method to probe Keplerian accretion disks near compact objects (particularly black holes) is through studying the profiles and variability of iron fluorescence lines (see Fabian et al. 2000; Reynolds 2000 for recent reviews). Furthermore, the new method provides a unique opportunity to check whether the neutron star is corotating with the disk, and potentially even to determine the misalignment angle between the spin axis and the disk symmetry axis. Such measurements can significantly enrich our knowledge of the origin and evolution of LMXBs.

The results of this paper demonstrate that the disk-scattered flux component manifests itself better in energy-resolved oscillation waveforms. Nearly coherent oscillations during type-I bursts, especially those detected during the burst rise, are probably the best candidates to look for signatures of disk-scattering. It is possible that RXTE data can already be used for such a search. For example, the phase lag of low-energy photons during a burst from Aql X-1 reported by Ford (1999) may be the result of Doppler boosting in a thin disk. It is important that this interpretation is self-consistent, as opposed to the alternative explanation based on Doppler boosting by the neutron star, as shown in Sect. 3.3. The case of the millisecond pulsar SAX J1808.4-3658, for which similar phase lags and

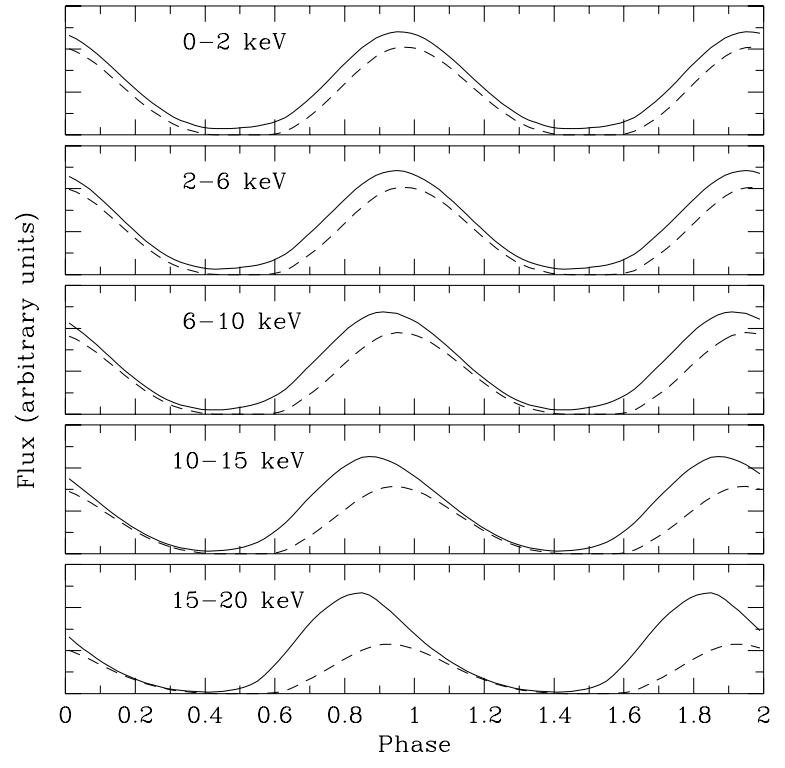

Fig. 10. Oscillation waveforms in five energy bands for the basic model with $i=60^{\circ}, \theta=90^{\circ}, f=300 \mathrm{~Hz}, \alpha=60^{\circ}$, the case of corotation and black-body spot emission with $k T=$ $2 \mathrm{keV}$. The solid lines are the total signal, and the dashed lines are the direct flux component.

also distortions of the pulse profile have been detected (Cui et al. 1998; Revnivtsev 1999; Ford 2000), appears to be more complicated. The principal difficulty here is that the observed energy spectrum has an approximately power-law shape. If this spectral distribution originates at the hot spot, then no energy dependence of the pulse profile can arise as a result of Doppler boosting (see, e.g., Chen \& Shaham 1989).

More detailed studies of the waveforms of X-ray flux oscillations in LMXBs could be possible with $\sim 6 \mathrm{~m}^{2}$ X-ray detectors (i.e., 10 times larger than RXTE), such as the recently proposed EXTRA and RAE missions (Barret et al. 2000; Kaaret et al. 2000; http://www.cesr.fr/ barret/extra.html). A particularly promising way to look for spin-phase signatures of disk-scattering, at least in the millisecond pulsar SAX J1808.4-3658, is by means of sensitive X-ray polarimetry. The case of SAX J1808.4-3658 (or similar objects which may be discovered in the future) is favourable because this source demonstrates regular pulsations during long time intervals, which allows one to accumulate a statistically large amount of data. On the contrary, such statistics is hardly achievable for nearly coherent burst oscillations, or for possible quasi-periodic oscillations originating in the spreading layer. Previously, only the time-integrated polarization due to Thomson scattering in the accretion disk (without allowance for its rotation) was discussed as an observational task for near-future X-ray polarimeters (Meszaros et al. 1988). An X-ray polarimeter will be part of the scientific payload of the Spectrum-X-Gamma observatory (Kaaret et al. 1993; http://hea-www.harvard.edu/ kaaret/sxrp/ index.html). Also, NASA is currently attaching great 


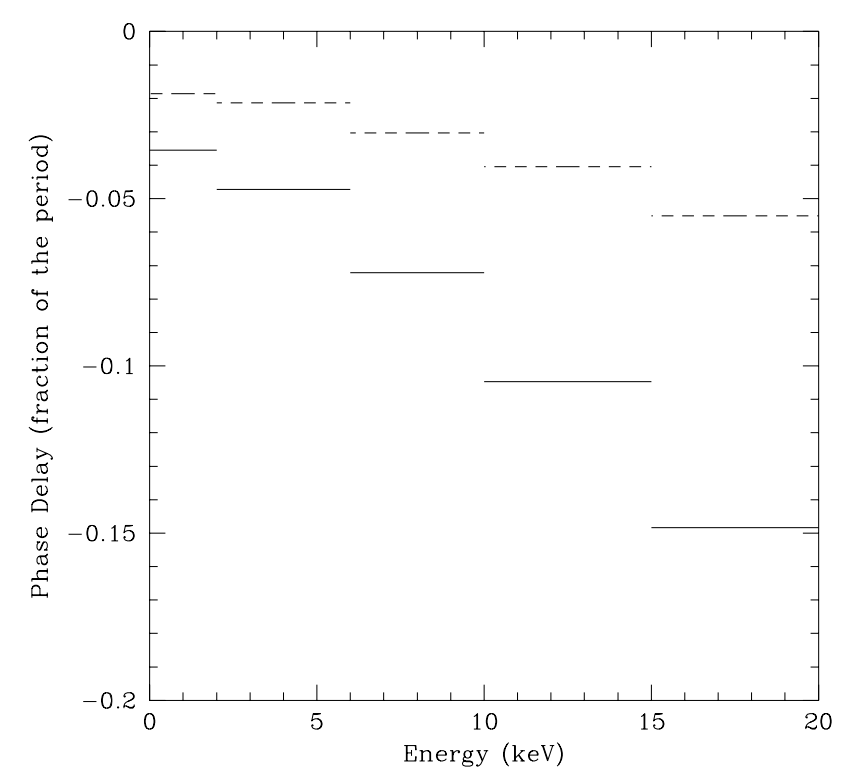

Fig. 11. Phase lag of the fundamental harmonic as a function of photon energy derived from the oscillation waveforms shown in Fig. 10. The solid bars correspond to the total signal, and the dashed bars to the direct flux component.

importance to a programme of long duration balloon flights, lasting up to 100 days (Jones 2000). This programme will no doubt rekindle interest in balloon flights of X-ray polarimeters.

\section{References}

Barret, D., Skinner, G. K., Staubert, R., \& Stella, L. 2000, Proc. 3rd Microquasar Workshop, ApSS, in press [astro-ph/0010632]

Basko, M. M., Sunyaev, R. A., \& Titarchuk, L. G. 1974, A\&A, 31,249

Chakrabarty, D., \& Morgan, E. H. 1998, Nature, 394, 346

Chandrasekhar, S. 1950, Radiative Transfer (Dover, New York)

Chen, K., \& Shaham, J. 1989, ApJ, 339, 279

Cui, W. C., Morgan, E. H., \& Titarchuk, L. G. 1998, ApJ, 504, L27

Fabian, A. C., Iwasawa, K., Reynolds, C. S., \& Young, A. J. 2000, PASP, 112, 1145

Ford, E. C. 1999, ApJ, 519, L73

Ford, E. C. 2000, ApJ, 535, L119
Galloway, D. K., Chakrabarty, D., Muno, M. P., \& Savov, P. 2001, ApJ, 549, L85

Gilfanov, M. R., Revnivtsev, M. G., Sunyaev, R. A., \& Churazov, E. M. 1998, A\&A, 338, L83

Inogamov, N. A., \& Sunyaev, R. A. 1999, Astron. Lett., 25, 269

Jones, W. V. 2000, Adv. Space Res., 26, 1303

Kaaret, P., et al. 1993, X-Ray and Ultraviolet Polarimetry, Proc. SPIE, 2010, 22

Kaaret, P., Grindlay, J., Lamb, F. K., et al. 2000, Astrophys. Lett. \& Comm., in press

Kluzniak, W., \& Wilson, J. R. 1991, ApJ, 372, L87

Landau, L. D., \& Lifshitz, E. M. 1975, The Classical Theory of Fields, 4th ed.: Landau and Lifshitz Course of Theoretical Physics (Pergamon press, Oxford)

Lapidus, I. I., \& Sunyaev, R. A. 1985, MNRAS, 217, 291

Meszaros, P., Novick, R., Chanan, G. A, Weisskopf, M. C., \& Szetgyorgyi, A. 1988, ApJ, 324, 1056

Miller, M. C., \& Lamb, F. K. 1993, ApJ, 413, L43

Narayan, R., Mahadevan, R., \& Quataert, E. 1998, in The Theory of Black Hole Accretion disks, ed. M. A. Abramowicz, G. Bjornsson, \& J. E. Pringleet (Cambridge Univ. Press, Cambridge), 148

Nayakshin, S., Kazanas, D., \& Kallman, T. 2000, ApJ, 537, 833

Pechenick, K. R., Ftaclas, C., \& Cohen, J. M. 1983, ApJ, 274, 846

Popham, R., \& Sunyaev, R. A. 2001, ApJ, 547, 355

Revnivtsev, M. G. 1999, Ph.D. Thesis, Space Research Institute, Moscow [astro-ph/9912556]

Reynolds, C. S. 2000, Proc. X-Ray Astronomy 1999, Bologna, Sep. 1999 [astro-ph/0001324]

Riffert, H., \& Meszaros, P. 1988, ApJ, 325, 207

Shakura, N. I., \& Sunyaev, R. A. 1973, A\&A, 24, 337

Sibgatullin, N. R., \& Sunyaev, R. A. 2000, Astron. Lett., 26, 699

Sobolev, V. V. 1949, Uchenye Zapisky Leningrad Univ., Ser. Mat. Nauk, 18, No. 16, in Russian

Strohmayer, T. E. 2000, Proc. X-Ray Astronomy 1999, Bologna, Sep. 1999 [astro-ph/9911338]

Sunyaev, R. A., \& Revnivtsev, M. G. 2000, A\&A, 358, 617

Van der Klis, M. 2000, ARA\&A, 38, 717

Weinberg, N., Miller, C. M., \& Lamb, D. Q. 2001, ApJ, 546, 1098

Wijnands, R., \& Van der Klis, M. 1998, Nature, 394, 344

Wijnands, R., Strohmayer, T., \& Franco, L. M. 2001, ApJ, 549, L71

Wood, K. S., Ftaclas, C., \& Kearney, M. 1988, ApJ, 324, L63 